\title{
ON THE POLAR DECOMPOSITION OF THE DUGGAL TRANSFORMATION AND RELATED RESULTS
}

\author{
SAJi Mathew And M. S. BAlasubramani
}

Abstract. Let $T=U|T|$ be the polar decomposition of a bounded operator $T$ on a Hilbert space. The transformation $\Delta(T)=|T|^{1 / 2} U|T|^{1 / 2}$ is called the Aluthe transformation, and $\Gamma(T)=|T| U$ is called the Duggal transformation of $T$. We discuss Aluthge transformation and Duggal transformation of binormal operators and centered operators. We obtain results about the polar decomposition of Duggal transformation. We give necessary and sufficient conditions for $\Gamma(T)$ to have the polar decomposition $\Gamma(T)=\Gamma(U)|\Gamma(T)|$. As a consequence we get $\Gamma(T)=$ $\Gamma(U)|\Gamma(T)|$ to be the polar decomposition of $\Gamma(T)$ if $T$ is binormal.

Mathematics subject classification (2000): 47A05, 47B20.

Keywords and phrases: Duggal transformation, Aluthge transformation, polar decomposition, binormal operators, centered operators.

\section{REFERENCES}

[1] A. Aluthge, On p-hyponormal operators for $0<p<1$, Integral Equations Operator Theory, 13:307-315, 1990.

[2] S. L. Campbell, Linear operators for which $T^{*} T$ and $T T *$ commute, Proc. Amer. Math. Soc., 34:177-180, 1972.

[3] X. Catepillan and W. Szymanski, Semigroup properties of factors in the polar decomposition or De Moivre's formula, Proc. Amer. Math. Soc., 126(12):3521-3526, 1998.

[4] C. Foias, I. B. Jung, E. Ko, And C. PEARCy, Complete contractivity of maps associated with the Aluthge and Duggal transforms., Pacific J. Math, 209(2):249-259, 2003.

[5] T. FURUTA, On the polar decomposition of an operator, Acta Sci. Math. (Szeged), 46:261-268, 1983.

[6] M. Ito, T. YAmaZAKI And M. YAnAGIDA, On the polar decomposition of the Aluthge transformation and related results, J. Operator Theory, 51:303-319, 2004.

[7] M. ITO, T. YAMAZAKI AND M. YANAGIDA, On the polar decomposition of the product of two operators and its applications, Integral Equations Operator Theory, 49(4):461-472, 2004.

[8] I. B. Jung, E. Ko AND C. PEARCY, Aluthge transforms of operators, Integral Equations Operator Theory, 37:437-448, 2000.

[9] B. B. Morrel And P. S. Muhly, Centered operators, Studia Math., 51:251-263, 1974. 